\title{
A MULTIVARIATE GENERALIZATION OF A THEOREM OF R. H. FARRELL
}

\author{
D. PLACHKY
}

(Communicated by Andrew M. Bruckner)

\begin{abstract}
Let $(\Omega, \mathfrak{S}, \mu)$ denote a finite measure space and $T_{j}: \Omega \rightarrow$ $\left[a_{j}, b_{j}\right]\left(\mathfrak{S}, \mathfrak{B} \cap\left[a_{j}, b_{j}\right]\right)$-measurable functions, $j=1, \ldots, n$. Then the algebra of functions generated by $1, T_{1}, \ldots, T_{n}$ is a dense subset of $\mathscr{L}_{1}(\Omega, \mathfrak{S}, P)$ if and only if for any $A \in \mathfrak{S}$ there exists some $B \in\left(T_{1}, \ldots, T_{n}\right)^{-1}\left(\mathfrak{B}^{n} \cap\right.$ $\left.\left[a_{1}, b_{1}\right] \times \cdots \times\left[a_{n}, b_{n}\right]\right)$, such that $\mu(A \triangle B)=0$ is valid. In particular, this condition is satisfied if $(\Omega, \mathfrak{S})$ is a Blackwell space and $\left(T_{1}, \ldots, T_{n}\right): \Omega \rightarrow$ $\left[a_{1}, b_{1}\right] \times \cdots \times\left[a_{n}, b_{n}\right]$ is in addition one-to-one.
\end{abstract}

If for any $A \in \mathfrak{S}$ there exists some

$$
B \in\left(T_{1}, \ldots, T_{n}\right)^{-1}\left(\mathfrak{B}^{n} \cap\left[a_{1}, b_{1}\right] \times \cdots \times\left[a_{n}, b_{n}\right]\right),
$$

where $\mathfrak{B}^{n}$ denotes the Borel $\sigma$-algebra of $\mathbb{R}^{n}$, such that $\mu(A \triangle B)=0$ holds, then for any $f \in \mathscr{L}_{1}(\Omega, \mathfrak{S}, \mu)$ there exists some Borel function $g:\left[a_{1}, b_{1}\right] \times$ $\cdots \times\left[a_{n}, b_{n}\right] \rightarrow \mathbb{R}$, such that $f=g \circ\left(T_{1}, \ldots, T_{n}\right)$ is valid a.e. $\mu$. Furthermore, any $g \in \mathscr{L}_{1}\left(\left[a_{1}, b_{1}\right] \times \cdots \times\left[a_{n}, b_{n}\right], \mathfrak{B}^{n} \cap\left[a_{1}, b_{1}\right] \times \cdots \times\left[a_{n}, b_{n}\right], \mu^{T}\right)$, where $T$ stands for $\left(T_{1}, \ldots, T_{n}\right)$, can be approximated by a continuous function $h:\left[a_{1}, b_{1}\right] \times \cdots \times\left[a_{n}, b_{n}\right] \rightarrow \mathbb{R}$ with respect to the $\mathscr{L}_{1}$-norm, and according to the approximation theorem of Bernstein any such function $h$ can be approximated uniformly by a polynomial of $\left(t_{1}, \ldots, t_{n}\right) \in\left[a_{1}, b_{1}\right] \times \cdots \times\left[a_{n}, b_{n}\right]$, which proves that the algebra of functions generated by $1, T_{1}, \ldots, T_{n}$ is dense in $\mathscr{L}_{1}(\Omega, \mathfrak{S}, \mu)$. Conversely, if this algebra of functions is a dense subset of $\mathscr{L}_{1}(\Omega, \mathfrak{S}, \mu)$, then any $f \in \mathscr{L}_{1}(\Omega, \mathfrak{S}, \mu)$ can be written as $f=$ $g \circ\left(T_{1}, \ldots, T_{n}\right)$ a.e. $\mu$ for some Borel function $g:\left[a_{1}, b_{1}\right] \times \cdots \times\left[a_{n}, b_{n}\right] \rightarrow$ $\mathbb{R}$, if one takes into consideration that $\mathscr{L}_{1}(\Omega, \mathfrak{S}, \mu)$-convergence implies $\mu$ stochastic convergence and any $\mu$-stochastic convergent sequence admits a subsequence that converges a.e. $\mu$. In particular, for any $A \in \mathfrak{S}$ there exists some $B \in T^{-1}\left(\mathfrak{B}^{n} \cap\left[a_{1}, b_{1}\right] \times \cdots \times\left[a_{n}, b_{n}\right]\right)$ satisfying $\mu(A \triangle B)=0$. Thus, the following has been proved:

Theorem. Let $(\Omega, \mathfrak{S}, \mu)$ denote a finite measure space and let $T_{j}: \Omega \rightarrow\left[a_{j}, b_{j}\right]$ be $\left(\mathfrak{S}, \mathfrak{B} \cap\left[a_{j}, b_{j}\right]\right)$-measurable, $j=1, \ldots, n$. Then the algebra of functions

Received by the editors March 12, 1990.

1980 Mathematics Subject Classification (1985 Revision). Primary 28A60, 28A25. 
generated by $1, T_{1}, \ldots, T_{n}$ is a dense subset of $\mathscr{L}_{1}(\Omega, \mathfrak{S}, \mu)$ if and only if for any $A \in \mathfrak{S}$ there exists some $B \in\left(T_{1}, \ldots, T_{n}\right)^{-1}\left(\mathfrak{B}^{n} \cap\left[a_{1}, b_{1}\right] \times \cdots \times\left[a_{n}, b_{n}\right]\right)$ satisfying $\mu(A \triangle B)=0$.

Example. If $(\Omega, \mathfrak{S})$ is a Blackwell space (cf. [3, p. 37]) and $\left(T_{1}, \ldots, T_{n}\right): \Omega \rightarrow$ $\left[a_{1}, b_{1}\right] \times \cdots \times\left[a_{n}, b_{n}\right]$ is a one-to-one Borel function, then according to a theorem of Blackwell $\mathfrak{S}=\left(T_{1}, \ldots, T_{n}\right)^{-1}\left(\mathfrak{B}^{n} \cap\left[a_{1}, b_{1}\right] \times \cdots \times\left[a_{n}, b_{n}\right]\right)$ holds (cf. [3, p. 38]); i.e., the algebra of functions generated by $1, T_{1}, \ldots, T_{n}$ is dense in $\mathscr{L}_{1}(\Omega, \mathfrak{S}, \mu)$. For $(\Omega, \mathfrak{S})=(\mathbb{R}, \mathfrak{B}), n=1$, and $T_{1}$ strictly monotone, this theorem is due to R. H. Farrell (cf. [2] and [5, p. 518]). It is not difficult to see that in this case $T_{1}^{-1}\left(\mathfrak{B} \cap\left[a_{1}, b_{1}\right]\right)=\mathfrak{B}$ is valid. This follows immediately from $[\alpha, \beta]=T_{1}^{-1}\left(\left[T_{1}(\alpha), T_{1}(\beta)\right]\right),[\alpha, \beta] \subset \mathbb{R}$ (if $T_{1}$ is strictly increasing), which implies $\mathfrak{B} \subset T_{1}^{-1}\left(\mathfrak{B} \cap\left[a_{1}, b_{1}\right]\right)$.

Remark. It might be interesting to mention that the condition that for all $A \in \mathfrak{S}$ there exists some $B \in \mathfrak{T}$, where $\mathfrak{T}$ denotes some sub- $\sigma$-algebra of $\mathfrak{S}$ satisfying $\mu(A \triangle B)=0$, is equivalent to the assertion that $\mu$ is an extremal point of the convex set $E$ consisting of all finite measures $\nu$ on $\mathfrak{S}$ satisfying $\nu|\mathfrak{T}=\mu| \mathfrak{T}$, where $\nu \mid \mathfrak{T}$ denotes the restriction of $\nu$ to $\mathfrak{T}$. If for $\nu \in E$ the approximation property holds and $\nu=\alpha \nu_{1}+(1-\alpha) \nu_{2}$ for some $\nu_{j} \in E, j=1,2,0<\alpha<1$, is valid, then $\nu=\nu_{1}=\nu_{2}$ follows if we choose for $A \in \mathfrak{S}$ some $B \in \mathfrak{T}$ with $\mu(A \triangle B)=0$. This implies $\nu_{j}(A \triangle B)=0, j=1,2$, which leads to $\nu_{j}(A)=\nu_{j}(B)=\mu(B)=\mu(A), j=1,2$; i.e., $\nu$ is an extremal point of $E$. For a functional analytical proof of the converse direction based on a Hahn-Banach type argument cf. [1] and [4]. For sake of completeness the following short, probabilistic proof based on the notion of conditional expectation is included:

Proof. Without loss of generality one can assume that the finite measure $\mu$ is a probability measure $P$. Then $P_{f}$ defined by

$$
P_{f}(A)=\int_{A} \frac{f}{E_{P}(f \mid \mathfrak{T})} d P, \quad A \in \mathfrak{S},
$$

where $E_{P}(f \mid \mathfrak{T})$ denotes the conditional expectation of $f, f: \Omega \rightarrow \mathbb{R} \mathfrak{S}$ measurable and $M \geq f \geq m>0$ for some $m, M \in \mathbb{R}$, belongs to $E$. Furthermore, $P_{f}(A)=\int_{A} g d P, A \in \mathfrak{S}$, is valid, where $g: \Omega \rightarrow \mathbb{R}$ is $\mathfrak{S}$-measurable and satisfies $0 \leq g \leq L$ a.e. $P$ for some $L \geq 1$. Hence, $h=(1-g) / L$ has the property $-1 \leq h \leq 1$ a.e. $P$ and $\int_{A} h d P=0, A \in \mathfrak{S}$. Now the assumption that some $P \in E$ is an extremal point of $E$ yields

$$
P(A)=\int_{A}(1+h) d P=\int_{A}(1-h) d P, \quad A \in \mathfrak{S},
$$

from which $\int_{A} h d P=0, A \in \mathfrak{S}$; i.e., $h=0$ a.e. $P$ follows. Therefore, $P_{f}=P$ holds, which leads for $f=\varepsilon+I_{A_{1}}, \varepsilon>0, A_{1} \in \mathfrak{S}$, to

$$
P(A)=\int_{A}\left(\frac{I_{A_{1}}}{E_{P}\left(I_{A_{1}} \mid \mathfrak{T}\right)} I_{\left\{E_{P}\left(I_{A} \mid \mathfrak{T}\right)>0\right\}}+I_{\left\{E_{P}\left(I_{A_{1}} \mid \mathfrak{T}\right)=0\right\}}\right) d P, \quad A \in \mathfrak{S},
$$


for $\varepsilon \rightarrow 0$. This implies $P\left(A_{1} \triangle\left\{E_{P}\left(I_{A_{1}} \mid \mathfrak{T}\right)>0\right\}\right)=0$; i.e., for any $A_{1} \in \mathfrak{S}$ there exists some $B_{1} \in \mathfrak{T}$, namely $B_{1}=\left\{E_{P}\left(I_{A_{1}} \mid \mathfrak{T}\right)>0\right\}$, satisfying $P\left(A_{1} \triangle B_{1}\right)$ $=0$.

\section{REFERENCES}

1. R. G. Douglas, On extremal measures and subspace density, II, Proc. Amer. Math. Soc. 17 (1966), 1363-1365.

2. R. H. Farrell, Dense algebras of functions in $L_{p}$, Proc. Amer. Math. Soc. 13 (1962), 324328.

3. P. A. Meyer, Probability and potentials, Blaisdell, Waltham, MA, 1966.

4. D. Plachky, Extremal and monogenic additive set functions, Proc. Amer. Math. Soc. 54 (1976), 193-196.

5. M. M. Rao, Measure theory and integration, Wiley, New York, 1987.

Institute of Mathematical Statistics, University of MÜnster, Einsteinstrasse 62, D4400 MÜNSTER, West GeRMANY 